\title{
A Concept for a High-Energy Gamma-ray Polarimeter
}

\author{
P. F. Bloser ${ }^{a}$, S. D. Hunter ${ }^{a}$, G. O. Depaola ${ }^{b}$, and F. Longo ${ }^{c}$ \\ ${ }^{a}$ NASA Goddard Space Flight Center, Code 661, Greenbelt, MD 20771, USA \\ ${ }^{b}$ Facultad de Matemática, Astronomía y Física, Universidad Nacional de Córdoba, Ciudad \\ Universitaria, 5000 Córdoba, Argentina \\ ${ }^{c}$ Department of Physics and INFN, via Valerio 2, I-34100 Trieste, Italy
}

\begin{abstract}
We present a concept for an imaging gamma-ray polarimeter operating from $\sim 50 \mathrm{MeV}$ to $\sim 1 \mathrm{GeV}$. Such an instrument would be valuable for the study of high-energy pulsars, active galactic nuclei, supernova remnants, and gamma-ray bursts. The concept makes use of pixelized gas micro-well detectors, under development at Goddard Space Flight Center, to record the electron-positron tracks from pair-production events in a large gas volume. Pixelized micro-well detectors have the potential to form large-volume 3-D track imagers with $\sim 100$ $\mu \mathrm{m}(\mathrm{rms})$ position resolution at moderate cost. The combination of high spatial resolution and a continuous low-density gas medium permits many thousands of measurements per radiation length, allowing the particle tracks to be imaged accurately before multiple scattering masks their original directions. The polarization of the incoming radiation may then be determined from the azimuthal distribution of the electron-positron pairs. We have performed Geant4 simulations of these processes to estimate the polarization sensitivity as a function of instrument parameters and event selection criteria.
\end{abstract}

Keywords: Polarimetry, Gamma-ray astronomy, pair production, gas detectors, Monte Carlo simulations

\section{INTRODUCTION}

Long recognized as a potentially valuable tool for high-energy astronomy, gamma-ray polarimetry is finally showing signs of becoming a viable observational technique. Traditional gamma-ray telescopes have thus far relied exclusively on imaging, spectroscopy, and timing to determine what physical processes are at work in high-energy sources. Due to low statistics, however, it is usually quite difficult to distinguish between competing physical models based on spectral and timing information alone. The promise of gamma-ray polarimetery stems from the fact that nearly all high-energy emission mechanisms can give rise to linearly polarized emission, though the polarization angle and degree of polarization are highly dependent on the source physics and geometry. ${ }^{1}$ Both synchrotron radiation, produced by relativisitic electrons spiraling around magnetic field lines, and curvature radiation, caused by electrons following field lines that are themselves tightly curved, produce linearly polarized radiation in which the angle traces the field direction and the degree is independent of energy. Compton scattering of photons off electrons, on the other hand, produces scattered radiation whose polarization degree depends on energy and scatter angle. These processes are expected to dominate the high-energy radiation of gammaray pulsars, gamma-ray bursts (GRBs), supernova remnants (SNRs), active galactic nuclei (AGN), and X-ray binaries (XRB). An especially exciting possibility is the radiation produced by magnetic photon splitting, which may play a role in pulsars and soft gamma-ray repeaters with particularly strong magnetic fields. The two photons produced should have strong polarizations in which the angles depend on energy. ${ }^{2}$ Thus gamma-ray polarization studies promise to provide a powerful new method for studying the physics of astronomical sources, just as polarization studies at optical and radio wavelengths have been doing for decades.

Despite this powerful scientific incentive, progress in the field of gamma-ray polarimetry has until recently been very slow. In principle, all three X-ray and gamma-ray interaction mechanisms - photoelectric absorption, Compton scattering, and pair production - are sensitive to the polarization vector of the incident photon: the

Further author information: (Send correspondence to P. F. Bloser)

P.F.B.: E-mail: bloser@milkyway.gsfc.nasa.gov 
secondary products of the initial interaction - the photoelectron, Compton-scattered photon and recoil electron, or electron-positron pair, respectively - are produced with momenta described by a $\cos ^{2} \theta$ angular distribution relative to the incident electric field (whether the maximum lies parallel or perpendicular to the field depends on the process). High-energy polarimeters, therefore, depend on measuring the azimuthal distribution of the secondary particles. Until recent years, technological constraints have prevented the accurate measurement of this distribution, severely limiting the polarization sensitivity of gamma-ray telescopes. Chief amongst these constraints were the insufficient position resolution of the detectors, which restricts the accuracy to which the azimuthal angles can be determined, the small number of readout channels possible with practical electronics designs, which limits how finely a position-sensing detector may be segmented, and the effects of multiple Coulomb scattering of the secondary charged particles in detector materials, which quickly distorts their initial momentum vectors. Due to these factors, the two largest gamma-ray telescopes ever flown in space, COMTPEL and EGRET onboard the Compton Gamma-Ray Observatory (CGRO), had negligible polarization sensitivity. ${ }^{1,3,4}$

This situation is finally changing for polarimeters based on Compton scattering and photoelectric absorption. In the case of Compton telescopes, major advances in detector position resolution, event reconstruction algorithms, and high-density readout electronics have allowed new designs that are compact, permitting a large effective area, while maintaining good angular resolution and background rejection. The IBIS instrument on INTEGRAL will have some polarization sensivitiy for this reason, ${ }^{5}$ and all currently discussed Advanced Compton Telescope (ACT) designs will naturally be highly sensitive to polarized radiation between several $100 \mathrm{keV}$ and a few MeV. ${ }^{6-10}$ Laboratory prototypes of dedicated Compton polarimeters have already demonstrated good sensitivity. ${ }^{11,12}$ Technological advances have also helped the cause of X-ray polarimetry using photoelectric absorption $(\sim 0.5-10 \mathrm{keV})$ through the emergence of micropattern gas detectors. These detectors combine a low-density gas medium with pixel readouts of very fine $(\sim 100 \mu \mathrm{m})$ pitch, so that the initial track of the photoelectron may be accurately measured before it is distorted by Coulomb scattering. Good sensitivity has been demonstrated in the laboratory for X-ray polarimeters using this principle. ${ }^{13,14}$ Thus polarimetry at X-ray and medium energy gamma-ray energies seems nearly ready to become reality. The recent detection via the Compton scattering technique of very strong $(\sim 80 \%)$ linear polarization from the prompt emission of a GRB $(0.15-2$ $\mathrm{MeV}$ ) by the RHESSI satellite ${ }^{15}$ has moved gamma-ray polarization to the forefront of high-energy astrophysical investigations and provided a major spur to the development of future missions.

The flurry of activity in gamma-ray polarimetry has thus far not extended to the pair production regime $(z 50 \mathrm{MeV})$. This is despite the fact that it is in practice far easier to construct sensitive pair telescopes than Compton telescopes due to better angular resolution, simpler event reconstruction, and lower background. The same technological advances that have enabled the development of photoelectric X-ray polarimeters, namely very fine spatial resolution in a gas detector, should in principle minimize the Coulomb scattering of the electronpositron pair and thus finally also allow the construction of a pair telescope with polarization sensitivity. In this paper we describe a concept for a high-energy gamma-ray polarimeter based on gas micro-well detectors, amorphous silicon thin film transistor arrays, and high-density interconnect and readout electronics technology. This design is a natural extension of a next-generation high-energy gamma-ray telescope concept introduced previously. ${ }^{16}$

\section{HIGH-ENERGY GAMMA-RAY POLARIMETRY}

\subsection{Basic Principles}

The idea of measuring high-energy ( $>50 \mathrm{MeV}$ ) gamma-ray polarization by detecting the asymmetric azimuthal distribution of the plane defined by the electron-positron pair has been discussed since the $1950 \mathrm{~s} .{ }^{17}$ Much theoretical work has been done since then to calculate the polarization-dependent cross section and estimate the detrimental effects of multiple Coulomb scattering. ${ }^{18-22}$ For the case where the incident photon, electron, and positron are nearly coplanar (i.e., the nuclear recoil is small), the azimuthal dependence of the cross section may be written ${ }^{18}$

$$
\sigma(\psi)=\frac{\sigma_{0}}{2 \pi}\left[1+P R \cos \left(2\left(\psi-\psi_{0}\right)\right)\right],
$$

where $\psi-\psi_{0}$ is the angle between the pair plane and the gamma ray's electric field vector, $\sigma_{0}$ is the total cross section, $P$ is the fractional polarization, and $R \sim 0.1$ is a numerical factor expressing the inherent asymmetry 
of the process. (More recent numerical calculations extend the cross section to non-coplanar pairs and suggest a more complex parameterization ${ }^{22}$; see Section 4.1.) In principle, then, one may search for polarized emission from a source by making a histogram of the recorded pair azimuth angles and fitting a function of the form $A \cos \left(2\left(\psi-\psi_{0}\right)\right)+B$. The strength of the azimuthal modulation may be expressed in terms of the modulation factor $Q$, usually defined as ${ }^{1,23}$

$$
Q=\frac{N_{\max }-N_{\min }}{N_{\max }+N_{\min }}=\frac{A}{B}
$$

where $N_{\max }$ and $N_{\min }$ are the number of counts recorded at the maximum and minimum of the distribution, respectively. In practice, rather than try to calculate $R$ in Equation 1, one would determine the modulation $Q_{100}$ resulting from $100 \%$ polarized radiation, either experimentally or by means of a Monte Carlo simulation. The degree of polarization measured from another source is then given simply by $P=Q_{\text {measured }} / Q_{100}$. More sophisticated statistical tests for the presence of azimuthal modulation have been developed as well ${ }^{24}$; the above basic formalism, however, yields a useful simple expression for the minimum detectable polarization (MDP) for a given instrument ${ }^{23}$ :

$$
M D P=\frac{n_{\sigma}}{Q_{100} S} \sqrt{\frac{2(S+B)}{T}} .
$$

Here $n_{\sigma}$ is the significance level, $S$ is the source count rate in the detector, $B$ is the background count rate, and $T$ is the observation time. The polarization sensitivity can therefore be improved by increasing the effective area, decreasing the background, or increasing $Q_{100}$.

The effects of Coulomb scattering may be approximated by replacing the factor $R$ in Equation 1 with

$$
R^{\prime}=R e^{-2 \Phi^{2}}
$$

where $\Phi \sim 14 L^{1 / 2}$ is the rms change in $\psi$ (assuming a Gaussian distribution) due to multiple scattering after passing through $L$ radiation lengths (RL) of material. ${ }^{4,20,24}$ (Monte Carlo simulations have found this approximation for $\Phi$ to be overly pessimistic, ${ }^{4,24}$ but it is still useful for comparing different telescope designs.) Thus the scattering has the effect of exponentially lowering $Q_{100}$, or exponentially worsening the MDP. This is the primary reason why all pair telescopes to date, as well as those currently planned, have negligible polarization sensitivity. For example, the EGRET telescope allowed gamma rays to convert into pairs in tantalum conversion foils $L=0.022 \mathrm{RL}$ thick. Although this gave the instrument a high efficiency, the resulting rms change in the pair azimuth in each foil was $\Phi=2.1$ radians. Thus $Q_{100}$ was reduced by a factor of $10^{-4}$, making EGRET insensitive to polarization from even the strongest cosmic sources. ${ }^{4,24}$ Currently planned instruments such as AGILE and GLAST use the same detection method of thick conversion foils, meaning that they too will not have a useful sensitivity to polarization.

\subsection{Advantages of a Gas Detector}

The design of a polarimeter with, for example, sensitivity $10^{3}$ times that of EGRET, i.e. with $R^{\prime} / R=10^{-1}$, requires, from Equation 4, that the direction of the electron and positron be determined after traversing a distance of $\sim 6 \times 10^{-3}$ RL. This distance, about one-fourth the thickness of one EGRET conversion foil, implies a track imaging detector with low density in terms of RL per sample measurement distance. A design for a next-generation high-energy gamma-ray telescope capable of arcminute single photon angular resolution requires a similar low-density track imager. ${ }^{16}$ It was found that arcminute resolution is achievable only above $\sim 3 \mathrm{GeV}$ and requires a track imaging detector with density less than $\sim 2 \times 10^{-5} \mathrm{RL}$ per sample. Using this design, the $6 \times 10^{-3} \mathrm{RL}$ would correspond to about 300 samples along the electron and positron tracks, more than sufficient to accurately determine the pair momenta. Such a low ratio of RL to sampling length can be achieved using gas detectors with very fine spatial resolution.

\section{ADVANCED PAIR TELESCOPE AND HIGH-ENERGY POLARIMETER DESIGN}

We have developed a concept for a high-energy gamma-ray telescope using gas micro-well detectors to achieve good polarization sensitivity. In this section we describe the detectors and readout electronics and present our concept for an advanced pair telescope (APT). The expected polarimetry performance is discussed in Section 4 . 

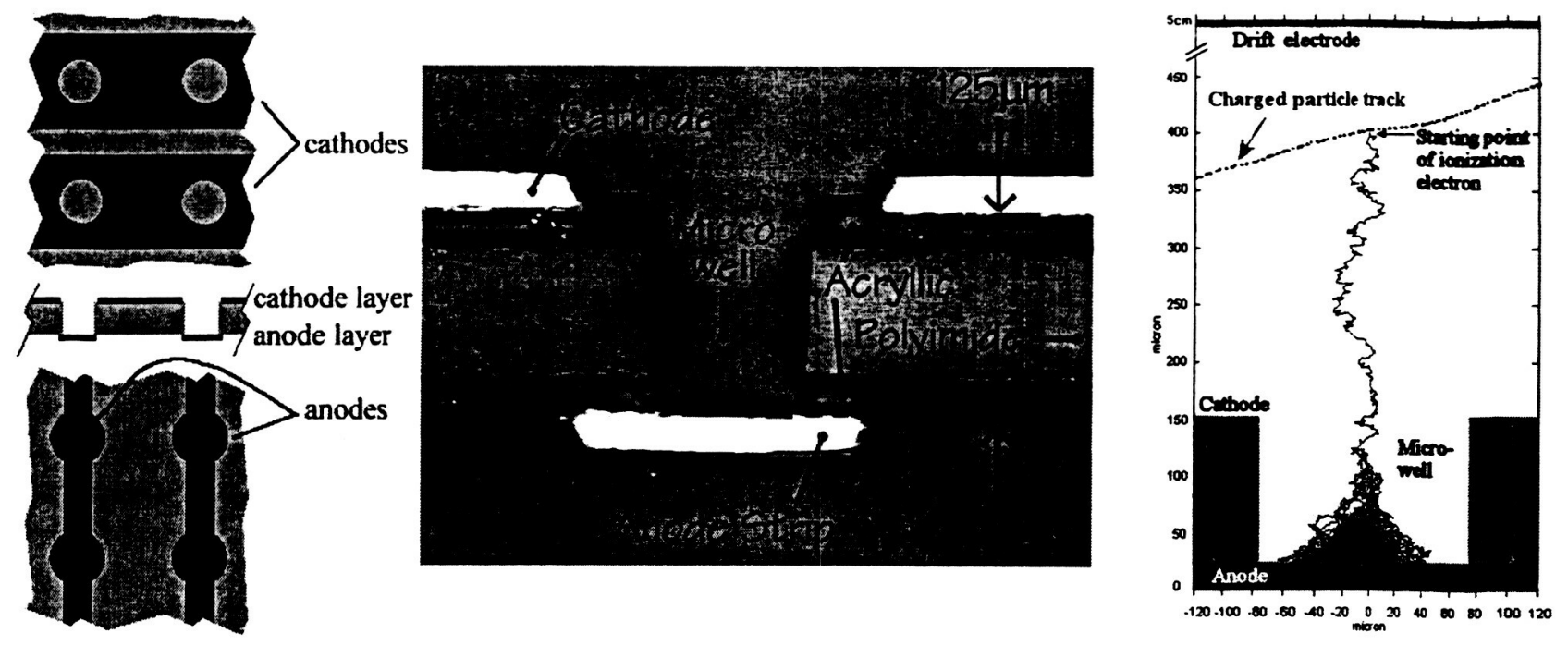

Figure 1. Micro-well detectors. Left: Copper cathode (top) and anode (bottom) traces for crossed-strip design. Wells are formed by exposing the anode through the cathode and substrate. Center: Cross section of an individual well formed by UV laser ablation with a polymide substrate. ${ }^{25,26}$ Right: Schematic of complete micro-well detector. The drift electrode defines the active region, in which fast charged particles generate ionization electrons which drift into the wells. Charge amplification takes place in the high-field avalanche region inside each well. The vertical scale has been greatly compressed.

\subsection{Pixelized Gas Micro-Well Detectors}

The micro-well detector ${ }^{25-27}$ is a type of gas proportional counter related to the gas electron multiplier. ${ }^{28}$ Each sensing element consists of a charge-amplifying well, as shown in Figure 1. The cathode and anode electrodes are deposited on opposite sides of an insulating substrate. The well is formed as a cylindrical hole through the cathode and substrate, exposing the anode. An array of such wells forms a detector. The active tracking volume is bounded by a drift electrode on one side and the wells on the other. Ionization electrons produced by the passage of a high-energy charged particle drift toward the anodes and into the wells. An ionization avalanche occurs in each well, where there is an intense electric field set up by the voltage applied between the anode and cathode. The electrons from the avalanche are collected on the anode, while an equal but opposite image charge is measured on the cathode.

Micro-well detectors have many advantages over conventional multi-wire proportional counters. The anodes and cathodes are rigidly affixed to the substrate, which simplifies construction and allows very fine pitch without electrostatic distortions. The detectors are very rugged and low cost, and the high intrinsic gain reduces the power required for readout electronics. The gain and the drift velocity are independently adjustable, allowing high gain to be combined with slow drift; this permits the detectors to be operated as time projection chambers. These properties make it practical to read out large gas volumes with very good three-dimensional position resolution at reasonable cost.

Goddard Space Flight Center has been producing micro-well detectors for the past several years on rugged polymide substrates using UV laser ablation. ${ }^{25,26}$ These detectors have been constructed in a crossed-strip geometry, with the cathodes and anodes forming orthogonal strips on opposite side of the substrate, to allow simple two-dimensional imaging at X-ray energies. Very good detector performance has been observed, ${ }^{25-27}$ including stable operation at gas gains of $3 \times 10^{4}$ in $\mathrm{Ar}$ - and $\mathrm{Xe}$ - based gases, the ability to sustain repeated breakdowns without damage, a FWHM energy resolution of $18 \%$ for $5.9 \mathrm{keV}$ X-rays in $95 \% \mathrm{Xe} / 5 \% \mathrm{CO}_{2}$ gas, and a FWHM position resolution of $\sim 200 \mu \mathrm{m}$ for $400 \mu \mathrm{m}$ well pitch.

The crossed-strip readout used for X-ray imaging is not ideal for recording extended tracks from charged particles. Thus we are currently focused on the development of pixelized micro-well detectors (PMWDs) together 

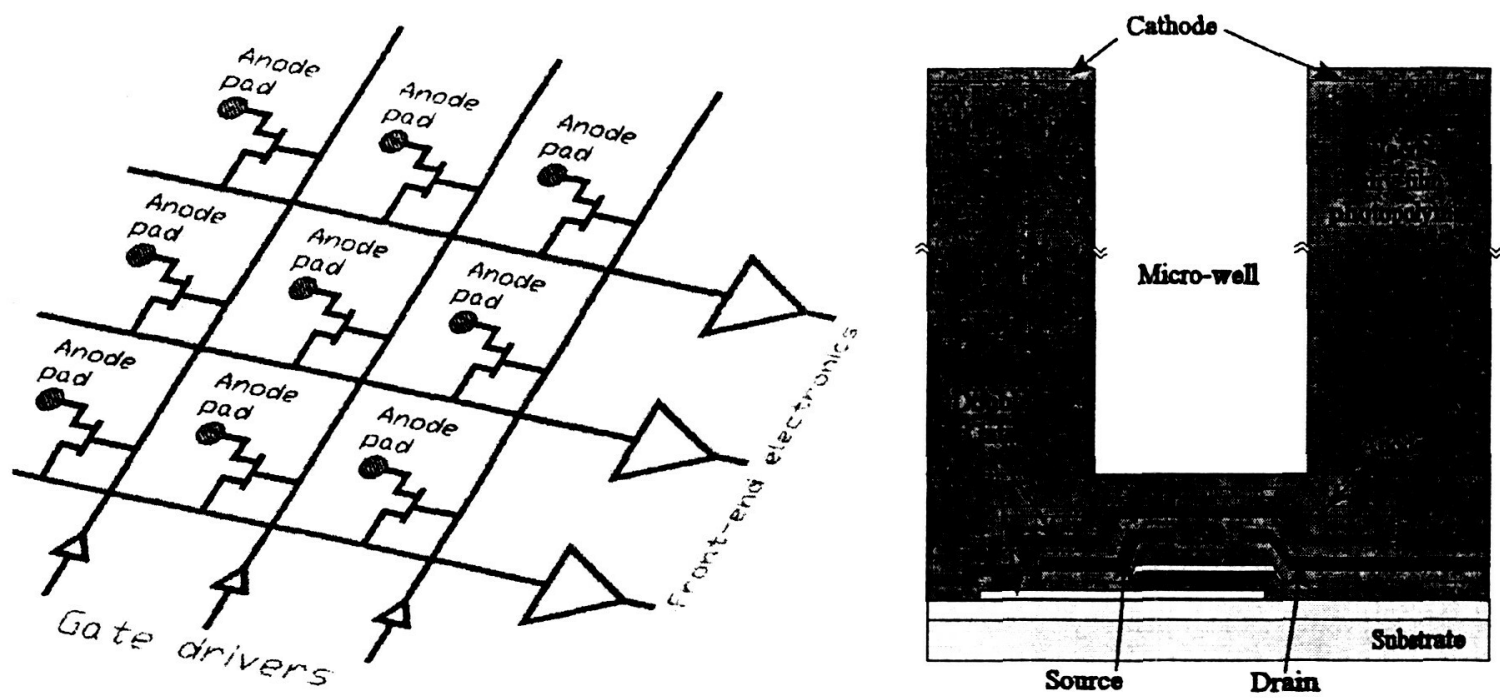

Figure 2. The pixelized micro-well detector concept. Left: Readout of a pixelized detector using a TFT array. The gate drivers are activated sequentially, allowing each column to be read out in turn by the charge-integrating front end electronics. Right: Cross section of a single integrated a-Si:H TFT/micro-well detector pixel. The drift electrode is not shown. The vertical scale is exaggerated.

with collaborators at the Pennsylvania State University (Figure 2). The anodes are segmented into individual pixel pads, each of which is connected to an element of a thin-film transistor (TFT) array. The individual transistor gates are connected in columns, and the outputs are connected in rows. The gate drivers for each column are then activated sequentially, allowing the charge collected on the anode pads to be read out by chargeintergating amplifiers at the end of each row. Thus a two-dimensional projected image of the charged particle track is recorded. The third dimension may be determined by measuring the drift time of the ionization electrons (see Section 3.2).

Our collaborators at Penn State have developed a tri-layer fabrication process for hydrogenated amorphous silicon (a-Si:H) TFTs and integrated TFT circuits on polyamide substrates. TFT arrays based on a-Si:H were first developed as drivers for flat-panel displays, and more recently as readout devices for medical images. ${ }^{29}$ The current TFT structure consists of a $500 \AA$ thick amorphous silicon active layer and $3000 \AA$ thick silicon nitride layers as the gate dielectric and passivation layers. The structure includes a double gate to reduce leakage current and prevent the FET from turning on due to the electic field of the micro-well avalanche. Good electical performance has been observed, with $I_{o n} / I_{\text {off }} \sim 10^{8}$ and low threshold voltage 0.8-2.5 V. Current work is focused on producing the micro-well detector and TFT arrays as one integrated unit (Figure 2) using $100 \mu \mathrm{m}$ thick-film photolithographic processing. The cathode and anodes are patterned on the film, and the wells are created by etching. Integrated PMWD-TFT detectors with a pitch of $200 \mu \mathrm{m}$ are currently being produced for testing.

The small pitch and large area of PMWDs imply a very high density for the readout electronics and interconnects. The front-end charge-integrating preamplifiers, gate drivers, and time-projection electronics will all require $\sim 50$ channels $\mathrm{cm}^{-1}$ for the detectors described above. This requires an ASIC implentation for the electronics. Two charge-integrating amplifiers in an ASIC package currently under consideration for the PMWDs are the IDEAS VA-SCM2 chip and the Indigo ISC9717 chip. We are also investigating tape-carrier packaging and tape automated bonding as means to achieve our high-density interconnects in a compact, modular manner appropriate for a space-flight instrument.

\subsection{Advanced Pair Telescope Concept}

The APT imager concept is driven by the requirement to achieve a density of less than $2 \times 10^{-5} \mathrm{RL}$ per sample (Section 2.2). The PMWD-TFT array detectors described above with $200 \mu \mathrm{m}$ well pitch (and corresponding 

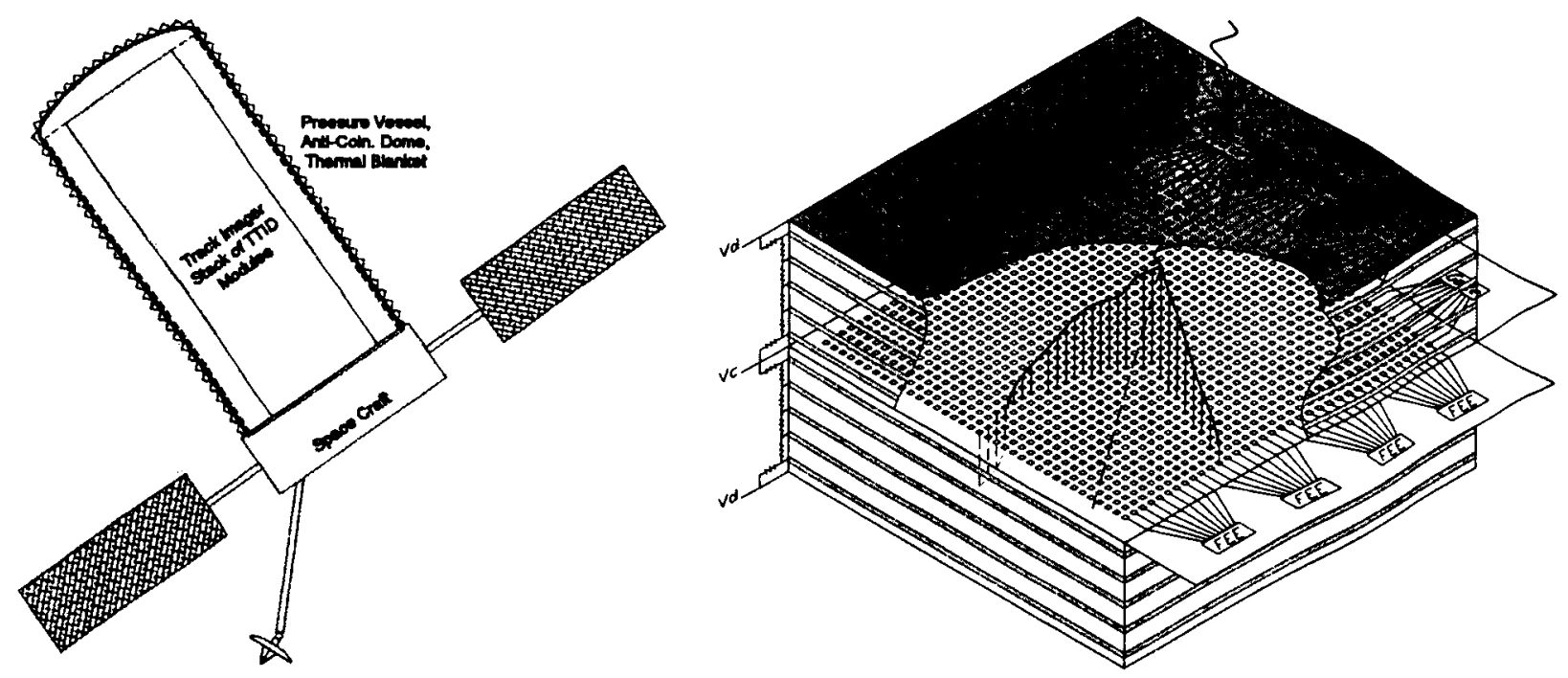

Figure 3. Left: Instrument concept for the Advanced Pair Telescope. Right: The TTID is based on pixelized micro-well detectors. Each individual TTID consists of a PMWD-TFT array (indicated by the array of squares), its front end electronics (FEE) and gate driver electronics (Gate), a drift electrode, and four support walls. The walls are ringed with field shaping electrodes. The APT instrument consists of a number of individual TTID back-to-back modules, illustrated here, stacked and arranged in layers, with the entire stack enclosed in a pressure vessel. The drift timing electronics (not shown) are located on the same circuit board as the gate driver electronics.

drift distance resolution) and a xenon gas mixture at a pressure of $P \sim 1.5$ atmospheres (RL $\sim 15 \times(1 \mathrm{~atm} / P)$ $\mathrm{m})$, achieves this density requirement. These PMWD-TFT arrays would be packaged into three-dimensional track imaging detector (TTID) modules. The APT track imager concept (Figure 3, left) consists of a large number of TTID modules (Figure 3, right). Each module contains two back-to-back imagers bounded by drift electrodes and field shaping electrodes on the four walls. The front-end, gate driver, and drift timing electronics are distributed around the periphery of the module and folded and attached to the walls of the module.

Photons undergo pair production in the active gas volume of the TTID, defined by the TFT array and the drift electrode. The electron and positron traverse the gas leaving a trail of ionization that drifts into the array of micro-wells. The electron and positron also excite the xenon gas producing UV scintillation light $(\lambda \sim 180$ $\mathrm{nm}$ ) that provides a prompt start signal for the drift timing and, combined with an anticoincidence detector, the event trigger.

The low density necessarily requires a large gas depth to obtain a reasonable pair conversion probability and instrument effective area. For xenon at $1.5 \mathrm{~atm}, 0.5 \mathrm{RL}$ (as in EGRET) corresponds to a depth of $5 \mathrm{~m}$. We therefore envision a pair telescope consisting of stacked layers of TTID modules in the form of a cylinder $5 \mathrm{~m}$ deep and $2.5 \mathrm{~m}$ in diamter. Modules in successive layers are rotated by $90^{\circ}$ so that the drift direction alternates, giving "stereo" projected images of the pair tracks. This give a redundant means of determining the three-dimensional paths of the tracks. Even though the gamma-ray incident direction and polarization angle are determined within about $6 \times 10^{-3} \mathrm{RL} \sim 6 \mathrm{~cm}$ of the vertex, the electron and positron continue to traverse the remainder of the gas. Since the entire depth of gas is instrumented, measuring the Coulomb scattering of the electron and positron can be used to determine their energy without the need for a massive calorimeter. ${ }^{16}$ It may also be possible to use an outer layer of gas as an anticoincidence layer, removing the need for a massive plastic shield dome.

\section{PREDICTED GAMMA-RAY POLARIMETER PERFORMANCE}

Preliminary estimates of the polarization sensitivity of the APT concept described in Section 3.2 have been made using Geant $4^{30}$ Monte Carlo simulations. These results should be considered tentative, given the experimental 


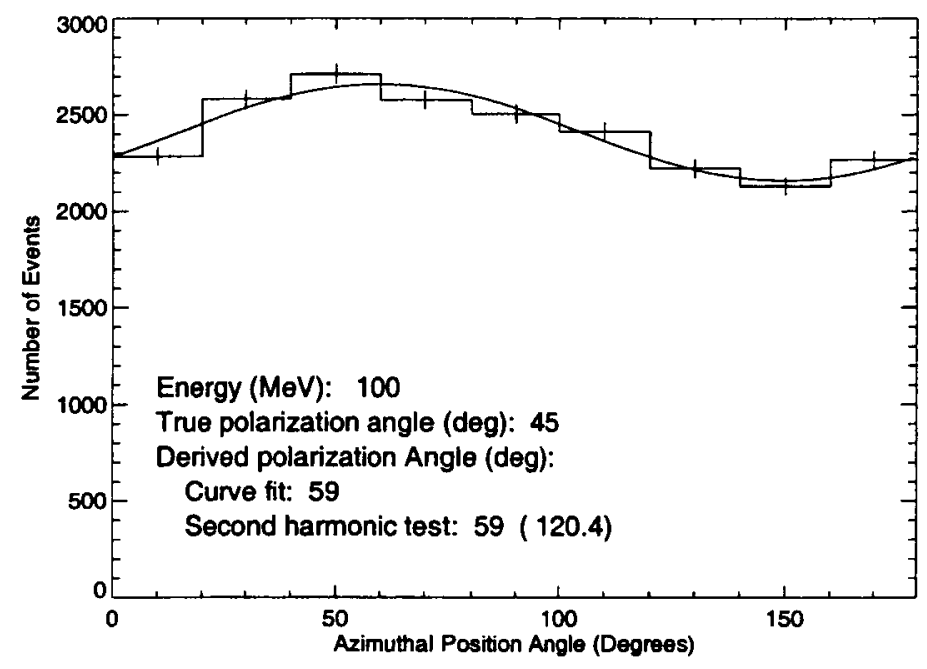

Figure 4. Output of the Geant4 polarized pair production process for $100 \mathrm{MeV}$ photons in xenon gas. The effects of Coulomb scattering and detector binning are not included. The derived polarization angle found by curve fitting is $59^{\circ}$, whereas the input angle was $45^{\circ}$. The modulation factor is $Q_{100}=0.104$. The second harmonic test also finds an angle of $59^{\circ}$; the value of the test statistic is given in parentheses.

nature of the Geant4 polarized pair production class.

\subsection{Geant4 Simulations of Polarized Pair Production}

Polarized pair production has been implemented in Geant4 by G. Depaola and F. Longo. ${ }^{22,31}$ The cross section for the pair production process, including the polarization of the incident photon and the effects of noncoplanarity, has been derived analytically and calculated numerically. Integrating over the energies and polar angles of the pair, this yielded the cross section as a function of the azimuthal offset from the polarization vector $\psi-\psi_{0}$ and the non-coplanarity angle $\phi$, defined as the angle between the electron and positron momenta projected into the plane normal to the incident photon (i.e., $\phi=\pi$ for coplanar events). A analytic parameterization of this surface is used for the Geant4 Monte Carlo sampling. Rather than the simple functional form of Equation 1 , the parameterization takes the form $f(\phi, \psi)=f_{\pi / 2}(\phi) \sin ^{2} \psi+f_{0}(\phi) \cos ^{2} \psi$, where $f_{\pi / 2}(\phi)$ and $f_{0}(\phi)$ are functions of $\phi$ whose coefficients are themselves functions of energy. The parameterization agrees with the numerically calculated surface to within $5 \%$.

Simulations were performed for the simple case of $100 \mathrm{MeV}$ gamma rays with a fixed polarization vector entering a xenon gas volume $5 \mathrm{~m}$ deep. No passive material was included in the mass model. During the tracking of the charged pair particles the step size was constrained to be $100 \mu \mathrm{m}$, or less than the pixel pitch, since otherwise energy was only deposited when a $\delta$-ray with energy greater than $1 \mathrm{keV}$ was generated. No detector response was included in the simulation; rather, the raw output (the exact positions of all energy deposits) was dumped into an output file, and the response of the detectors was applied in a separate program. This response included the effects of electron diffusion in the gas, binning into well pixels, and the expected error in the drift distance (assumed to be the same as the well pitch). Finally a simple event reconstruction algorithm was applied: each of the two tracks was fit with a straight line for as long a distance as possible, and this was used to determine the infall direction and azimuth angle of the pair plane. Energy-weighting of the reconstructed photon direction is not yet implemented.

Figure 4 shows the output of the Geant4 pair polarization process. Events were selected from the raw event file, taking the exact positions of all energy deposits and using only the first two hits of the electron and positron tracks (100 $\mu \mathrm{m}$ from the vertex) to define the initial pair momenta. Thus the effects of Coulomb scattering and detector binning were removed and only the effect of the parameterized cross section should be present. Only 
events falling within an angular radius containing $68 \%$ of all events were included. The input polarization angle was $45^{\circ}$.

The presence of an azimuthal modulations was searched for in two ways. The first is a simple function fitting as described in Section 2.1. This yielded a polarization angle of $\psi_{0}=59^{\circ}$ and a modulation factor $Q_{100}=0.104$. The second method is the "second harmonic test" used to search for polarization signals in COS B and EGRET data. $^{4,24}$ The value of the test statistic must exceed 13.8 for a $3 \sigma$ detection of azimuthal asymmetry. The test statistic for this dataset, given in parentheses in Figure 4, is 120.4, a very significant detection. The polarization angle is found to be $59^{\circ}$ for this test as well. This shift in the polarization angle appears to be due to a warping in the parameterized cross section surface, in which the minimum shifts to higher $\psi$ as $\phi$ decreases. The cause of this warping is not yet understood.

\subsection{Expected Polarization Sensitivity \\ NO RESULTS YET.}

\section{ACKNOWLEDGMENTS}

This work was performed while the author held a National Research Council Research Associateship Award at NASA/GSFC.

\section{REFERENCES}

1. F. Lei, A. J. Dean, and G. L. Hills, "Compton polarimetry in gamma-ray astronomy," Space Sci. Rev. 82, pp. 309-388, 1997.

2. M. Baring, "Photon splitting limits to the hardness of emission in strongly magnetized soft gamma repeaters," Astrophys. Jour. 440, pp. L69-L72, 1995.

3. F. Lei, G. L. Hills, A. J. Dean, and B. M. Swinyard, "Characteristics of COMPTEL as a polarimeter and its data analysis," Astrophys. Jour. Supp. Ser. 120, pp. 695-698, 1996.

4. J. R. Mattox, "The sensitivity of EGRET to gamma-ray polarization," Exp. Astron. 2, pp. 75-84, 1991.

5. J. B. Stephen, E. Caroli, R. C. da Silva, and L. Foschini, "Gamma-ray polarisation measurements with INTEGRAL/IBIS," in Gamma 2001, S. Ritz, N. Gehrels, and C. R. Shrader, eds., AIP Conf. Proc. 587, pp. 816-820, 2001.

6. T. J. O'Neill and et al., "Tracking, imaging and polarimeter properties of the TIGRE instrument," Astron. Astrophys. Supp. Ser. 120, pp. 661-664, 1996.

7. G. Kanbach, R. Andritschke, P. F. Bloser, F. Schopper, V. Schonfelder, and A. Zoglauer, "Concept study for the next generation medium-energy gamma-ray astronomy mission - MEGA," in X-Ray and GammaRayTelescopes and Instruments for Astronomy, J. E. Truemper and H. D. Tananbaum, eds., Proc. SPIE 4851, pp. 1209-1220, 2003.

8. E. Aprile, A. Bolotnikov, D. Chen, R. Mukherjee, and F. Xu, "The polarization sensitivity of the liquid xenon imaging telescope," Astrophys. Jour. Supp. Ser. 92, pp. 689-692, 1994.

9. S. E. Boggs and et al., "Upcoming balloon flight of the nuclear Compton telescope," in X-Ray and GammaRay Telescopes and Instruments for Astronomy, J. E. Truemper and H. D. Tananbaum, eds., Proc. SPIE 4851, pp. 1221-1227, 2003.

10. J. D. Jurfess and R. A. Kroeger, "Progress towards an advanced Compton telescope," in Gamma 2001, S. Ritz, N. Gehrels, and C. R. Shrader, eds., AIP Conf. Proc. 587, pp. 867-876, 2001.

11. M. L. McConnell, J. R. Ledoux, J. R. Macri, and J. M. Ryan, "A hard X-ray polarimeter for gammaray bursts and solar flares," in X-Ray and Gamma-Ray Telescopes and Instruments for Astronomy, J. E. Truemper and H. D. Tananbaum, eds., Proc. SPIE 4851, pp. 1382-1393, 2003.

12. R. A. Kroeger, W. N. Johnson, J. D. Kurfess, and B. F. Phlips, "Gamma-ray polarimetry using a position sensitive germanium detector," Nucl. Inst. Meth. A 436, pp. 165-169, 1999.

13. E. Costa, P. Soffitta, R. Bellazzini, A. Brez, N. Lumb, and G. Spandre, "An efficient photoelectric X-ray polarimeter for the study of black holes and neutron stars," Nature 411, pp. 662-665, 2001. 
14. J. K. Black, P. Deines-Jones, S. E. Ready, and R. A. Street, "X-ray polarimetry with an active-matrix pixel proportional counter," Nucl. Inst. Meth. A, in press.

15. W. Coburn and S. E. Boggs, "Polarization of the prompt $\gamma$-ray emission from the $\gamma$-ray burst of 6 December 2002," Nature 423, pp. 415-417, 2003.

16. S. D. Hunter, D. L. Bertsch, and P. Deines-Jones, "Design of a next generation high-energy gamma-ray telescope," in Gamma 2001, S. Ritz, N. Gehrels, and C. R. Shrader, eds., AIP Conf. Proc. 587, pp. 848$852,2001$.

17. G. C. Wick, "Detection of gamma-ray polarization by pair production," Phys. Rev. 81, pp. 467-468, 1951.

18. L. C. Maximon and H. Olsen, "Measurement of linear photon polarization by pair production," Phys. Rev. 126, pp. 310-319, 1962.

19. S. R. Kel'ner, Y. D. Kotov, and V. M. Logunov, "Methods of measuring linear polarization of gamma quanta," Sov. Jour. Nucl. Phys. 21, pp. 313-315, 1975.

20. A. A. Kozlenkov and I. G. Mitrofanov, "Polarimetry of cosmic sources of hard gamma-ray emission," Sov. Astron. 29, pp. 591-593, 1985.

21. Y. D. Kotov, "Methods of measurement of gamma-ray polarization," Space Sci. Rev. 49, pp. 185-195, 1988.

22. G. O. Depaola, C. N. Kozameh, and M. H. Tiglio, "A method to determine the polarization of high energy gamma rays," Astropart. Phys. 10, pp. 175-183, 1999.

23. R. Novick, "Steller and solar X-ray polarimetry," Space Sci. Rev. 18, pp. 389-408, 1975.

24. J. R. Mattox, H. A. Mayer-Hasselwander, and A. W. Strong, "Analysis of COS B data for evidence of linear polarization of Vela pulsar gamma rays," Astrophys. Jour. 363, pp. 270-273, 1990.

25. J. K. Black, P. Deines-Jones, S. D. Hunter, and K. Jahoda, "Imaging micro-well detectors for X-ray and gamma-ray applications," in X-ray and Gamma-ray Instrumentation for Astronomy XI, K. A. Flanagan and O. H. W. Siegmund, eds., Proc. SPIE 4140, pp. 313-323, 2000.

26. P. Deines-Jones, J. K. Black, H. Crawford, and S. D. Hunter, "Imaging micro-well proportional counters fabricated with masked UV laser ablation," Nucl. Inst. Meth. A 477, pp. 55-58, 2002.

27. P. Deines-Jones, J. K. Black, S. D. Hunter, K. Jahoda, and S. M. Owens, "Large-area imaging micro-well detectors for high-energy astrophysics," Nucl. Inst. Meth. A 478, pp. 130-134, 2002.

28. F. Sauli, "GEM: a new concept for electron amplification in gas detectors," Nucl. Inst. Meth. A 386, pp. 531-534, 1997.

29. L. E. Antonuk and et al., "Large-area $97-\mathrm{mm}$ pitch indirect-detection active-matrix flat-panel imager (AMFPI)," Proc. SPIE 3336, pp. 2-13, 1998.

30. S. Agostinelli and et al., "Geant4 - a simulation toolkit," Nucl. Inst. Meth. A 506, pp. 250-303, 2003.

31. G. O. Depaola, "Azimuthal distribution for pair production by high-energy $\gamma$-rays," Nucl. Inst. Meth. A 452, pp. 298-305, 2000. 